\title{
Association of NOD2/CARD15 mutations with previous surgical procedures in Crohn's disease
}

\author{
M. Barreiro, C. Núñez ${ }^{1}$ J. E. Domínguez-Muñoz, A. Lorenzo, F. Barreiro², J. Potel ${ }^{2}$ and A. S. Peña ${ }^{1,3}$

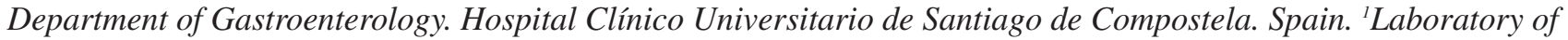 \\ Immunogenetics. Vrije Universiteit Medish Centrum. Amsterdam, The Netherlands. ${ }^{2}$ Department of Surgery. Hospital \\ Clínico Universitario. Santiago de Compostela. A Coruña, Spain. ${ }^{3}$ Department of Gastroenterology. Vrije Universiteit \\ Medish Centrum. Amsterdam, The Netherlands
}

\begin{abstract}
Objective: the aim of this study is to assess the importance of NOD2/CARD15 gene mutations as prognostic factors for surgical indications in Crohn's disease.

Patients and experimental design: a total of 165 Crohn's disease patients were studied, considering previous surgery related to Crohn's disease. We analyzed for previous surgery in global procedures as well as separately for the two main surgical indications: ileal resection and fistula treatment. The need for appendectomy was also studied. All patients were genotyped for the three CARD15 mutations, and association studies were developed using Chi-square statistics and Fisher's exact test whenever appropriate.

Results: carriers of the G908R or $1007 \mathrm{fs}$ mutation needed surgery more frequently, both for ileal resection and fistula repair. In contrast, appendectomy was not associated with CARD15 mutations.

Conclusions: as previously reported in this population, the R702W mutation does influence parameters of disease or need of surgery. The need for Crohn's disease-related surgery is higher in carriers of the G908R or $1007 \mathrm{fs}$ CARD15 mutation in the Galician population. Nevertheless, the frequency of these mutations does not allow their use to predict the course of disease.
\end{abstract}

Key words: NOD2/CARD15. Crohn's disease. Phenotypes. Surgery.

Barreiro M, Núñez C, Domínguez-Muñoz JE, Lorenzo A, Barreiro F, Potel J, Peña AS. Association of NOD2/CARD15 mutations with previous surgical procedures in Crohn's disease. Rev Esp Enferm Dig 2005; 97: 547-553.

\footnotetext{
Manuel Barreiro has received a grant from IDICHUS Foundation; Concepción Núñez received a scholarship from Ramón Areces Foundation, Spain, to spend two years in the Laboratory of Immunogenetics at VUMC Amsterdam, The Netherlands
}

Recibido: 16-07-04.

Aceptado: 01-02-05.

Correspondencia: Manuel Barreiro de Acosta. C/ San Pedro de Mezonzo, 38, P 6º 15701 Santiago de Compostela. A Coruña. Fax: 981951378. e-mail: manubarreiro@hotmail.com

\section{INTRODUCTION}

The etiology of Crohn's disease (CD) is still unknown. However, there is consensus that an inappropriate mucosal immune response to normal constituents of the intestinal microflora in genetically susceptible individuals is important in the pathogenesis. Although several genes have been involved in the susceptibility of this disease $(1,2)$, the CARD15 gene, previously called the NOD2 gene, is the main susceptibility gene to CD in most studied populations $(1,3)$. The CARD15 gene is located in the pericentromeric region of chromosome 16 (16q12) and codes for a NOD2 protein, which acts as an intracellular receptor for bacterial components, specifically peptidoglycans. There are three functional mutations of the CARD15 gene, two missense variants and one deletion: R702W, G908R and 1007fs, respectively. However, these mutations are not present in all the populations studied so far. Important differences in the frequency of these mutations have been observed according to each population's race and geographic location (4-13). In most of the studies reported, the presence of CARD15 mutations has been associated with ileal disease, which usually implies a need for surgery with higher frequency.

Recently, we reported a study carried out in the Galician population (13). CD was significantly associated with G908R and 1007fs mutations, although with a smaller frequency versus the majority of Caucasian populations. CD patients require surgery at some point during their lives, with ileal resection and fistula repair being the most frequent procedures. It is noteworthy that a high number of these patients also needed appendectomy, previously to or at the time of diagnosis. However, the relationship of these three main mutations of the CARD15 gene with CD-related surgery has not been specifically studied as far as we know.

The aim of our work was to assess the influence of CARD15 mutations on the various CD-related surgical 
procedures, and to determine whether these mutations may be used to predict need of surgery.

\section{METHODS}

\section{Patients and controls}

A total of 165 patients with Crohn's disease followed up in the Inflammatory Bowel Disease Unit, Gastroenterology Department, at Hospital Clínico Universitario, Santiago de Compostela were studied. All patients were Galician and residents of Galicia. As controls, a group of 165 healthy, unrelated, ethnically-matched individuals with no family history of IBD and with similar characteristics regarding age, sex and origin were chosen (controls were also Galician and residents of Galicia). From each subject a blood sample in EDTA tubes was obtained by venipuncture with the corresponding written informed consent. The hospital ethical committee approved the protocol.

The diagnosis of CD was made by clinical, radiological, endoscopic, and histological investigations. The patients and their data were reviewed by the same gastroenterologist to confirm the diagnosis and to avoid different criteria for diagnosis establishment. A retrospective study of patients regarding previous need for CD-related surgery was performed in collaboration with the Surgery Department of the same Hospital. The analysis was also stratified according to the two most frequent procedures in these patients, namely ileal resection and fistula (mainly perianal) repair. The history of appendectomy before the development of the disease or at the time of diagnosis was also analyzed.

\section{Determination of CARD15 mutations}

As previously published, genomic DNA was isolated from blood samples obtained from each individual using a Roche DNA isolation kit (Roche Molecular Biochemicals, Mannheim, Germany). All study participants were genotyped for the three mutations described as involved in CD (R702W, G908R and 1007fs) by means of PCRRFLP. Restriction fragments were isolated on $2 \%$ (G908R) or 4\% (R702W and 1007fs) agarose gels and visualized with ethidium bromide. Primers used to assay the R702W polymorphism included:

5'CGCACAACCTTCAGATCACA3' (forward) and

5'GGATGGAGTGGAAGTGCTTG3' (reverse).

For the G908R variant:

5'AAGTCTGTAATGTAAAGCCAC3' (forward) and

5'CCCAGCTCCTCCCTCTTC3' (reverse),

And the primers used for the 1007 fs polymorphism included:

5'GGCAGAAGCCCTCCTGCAGGGCC3' (forward) and

5'CCTCAAAATTCTGCCATTCC3' (reverse).
The association of the various mutations with $\mathrm{CD}$, overall or each specific subphenotype, was studied employing Chi-square tests or Fisher's exact test when necessary. Analyses were performed considering the influence of each specific mutation on $\mathrm{CD}$ as well as the influence of carrying more than one mutation. $\mathrm{P}$ values $<0.05$ were considered significant. Statistical analyses were performed using the SPSS software v. 11.0. This study has been approved by the Ethical Committee for Clinical Investigation of Galicia (2002/151).

\section{RESULTS}

All 165 consecutive patients included in this study were followed up for a mean of 8 years since the diagnosis of disease, with a range of 1-33 years. Mean age was 36 years, with a range between 17 and 76 . A slight predominance in women was seen $(57.6 \%)$.

A total of 85 patients $(51.5 \%)$ underwent a surgical procedure related to $\mathrm{CD}$. Among them, 51 patients $(60 \%)$ had needed an ileal resection, sometimes more than once, and 19 patients $(22.4 \%)$ had been treated for fistulae; 32 patients $(19.4 \%)$ had been appendectomized before or at the time of $\mathrm{CD}$ diagnosis.

In all, 27.9\% of patients carried at least one of the CARD15 mutations previously associated with CD. An R702W mutation was observed in 22 patients (13.3\%), G908R in $13(7.9 \%)$ and 1007 fs was observed in 14 patients $(8.5 \%)$. The $\mathrm{R} 702 \mathrm{~W}$ mutation showed also a relatively high frequency (11.5\%) in the Galician subjects without $\mathrm{CD}$ used as controls, similar to a previously reported study in another population (13). Therefore, this mutation does not seem to be involved in CD susceptibility.

The overall analysis of results showed that $39.1 \%$ of patients operated for $\mathrm{CD}$ (fistulae or ileal resection) showed at least one of the three main mutations in CARD15 vs. $19.8 \%$ of surgery-naïve CD patients. Differences between both groups were statistically significant $(\mathrm{p}<0.01)$. Considering only individuals carrying the two mutations previously associated with $\mathrm{CD}$ in the Galician population, G908R and $1007 \mathrm{fs}, 27.5 \%$ of them had undergone surgery, which is in contrast to only $6.3 \%$ who had not been operated $(\mathrm{p}<0.001)$. A more detailed analysis of the surgical procedures performed showed that CARD15 mutations were significantly more frequent in patients having undergone ileal resection $(43.1 \%)$ than in those without ileal resection $(21.1 \%)(\mathrm{p}=0.003)$. Statistically significant differences were also observed when individuals carrying only G908R and 1007 fs were considered, $29.4 v s$. $8.8 \%$ of carriers who had or had not undergone surgery $(p=0.001)$. Patients treated for fistulae had a higher frequency of CARD15 mutations, but differences were only significant when patients carrying both mutations were considered; $29.4 \%$ of the patients operated on for fistulae carried G908R and 1007fs vs. $8.8 \%$ of patients not operated on $(\mathrm{p}<0.01)$. 
The individual analysis of each mutation showed that the R702W mutation has approximately the same frequency in patients operated or otherwise (13.0 vs. $13.5 \%$, respectively). The G908R mutation showed a statistically significant higher frequency in previously operated patients $(14.5 \%)$ versus non-operated patients $(3.1 \%)$. The insertion mutation (1007fs) was observed in $14.5 \%$ of operated patients and in $4.2 \%$ of non-operated patients $(\mathrm{p}<$ 0.05 ). The frequency of each mutation regarding the different kind of surgery considered in this study is shown in table I. When the results of each surgical indication were analyzed individually, we found that carriers of the G908R mutation showed a significantly higher frequency of fistula treatments (31.6 vs. 4.8\%; p < 0.01). Regarding ileal resections, the $1007 \mathrm{fs}$ variant was found to be significantly associated with this kind of surgery $(17.6 \%$ of ileal resection patients carried this mutation vs. $4.4 \%$ of patients not having undergone this operation $\mathrm{p}<0.05)$. The G908R variant was also more frequent in patients with fistula repair $(13.7 v s .5 .3 \%)$, but differences were not significant $(\mathrm{p}<0.1)$.

An association was found between CARD15 mutations and appendectomy. However, the frequency of CARD15 mutations was higher in patients who had not been appendectomized.

Table I. Frequencies (\%) for carriers of CARD15 variants in relation to each surgery studied

\begin{tabular}{|c|c|c|c|c|c|c|c|}
\hline & & \multicolumn{2}{|c|}{ R702W } & \multicolumn{2}{|c|}{ G908R } & \multicolumn{2}{|c|}{$1007 f s$} \\
\hline lleal surgery & $\begin{array}{l}\text { Yes } \\
\text { No }\end{array}$ & $\begin{array}{l}15.7 \\
12.3\end{array}$ & $p=0.55$ & $\begin{array}{r}13.7 \\
5.3\end{array}$ & $p=0.06$ & $\begin{array}{r}17.6 \\
4.4\end{array}$ & $p=0.01$ \\
\hline Fístula surgery & $\begin{array}{l}\text { Yes } \\
\text { No }\end{array}$ & $\begin{array}{r}5.3 \\
14.4\end{array}$ & $p=0.27$ & $\begin{array}{l}31.6 \\
4.8\end{array}$ & $p=0.001$ & $\begin{array}{c}0 \\
9.6\end{array}$ & $p=0.16$ \\
\hline Appendectomy & $\begin{array}{l}\text { Yes } \\
\text { No }\end{array}$ & $\begin{array}{r}12.5 \\
13.5\end{array}$ & $p=0.88$ & $\begin{array}{c}0 \\
9.8\end{array}$ & $p=0.07$ & $\begin{array}{l}6.3 \\
9.0\end{array}$ & $p=0.61$ \\
\hline
\end{tabular}

Frequencies are expressed as percentages.

\section{DISCUSSION}

Previous studies have found that around $70-80 \%$ of $\mathrm{CD}$ patients require $\mathrm{CD}$-related surgery at least once during their lives -often more than once (14). Although predictive factors for the need of CD-related surgery are not entirely understood, patients with an ileal location and stricturing pattern need surgery more frequently. In the population we analyzed in this study, $51.5 \%$ of patients needed CD-related surgery at least once, although this frequency is lower than that previously described. This is probably due to the fact that our cohort is relatively young, with a mean 7.5 years of follow-up, and with an important number of patients with less than 4 years of disease.

The number of patients carrying at least one of the three CARD15 mutations in the Galician population $(27.9 \%)$, although lower than in most European populations $(5,11,12,15-17)$, permits to evaluate the relationship between these mutations and CD-related surgery.

First of all, appendectomy is not significantly associated with CARD15 mutations. However, it is noteworthy that in all cases a higher frequency of mutations in patients who have not experimented appendectomy is observed. It is known that appendectomy has a protective role against the development of UC. Results are still controversial in CD but it seems that patients needing appendectomy at onset of disease show a more severe course than patients who had previously experienced appendectomy. This suggests a protective role for the disease. It has been previously observed that appendectomy may delay the onset of CD $(18,19)$. Therefore, the results observed in this study may result from a preventive role of appendectomy in patients with susceptibility genes other than CARD 15 regarding disease development.

Regarding CD-related surgery, it appears that CARD15 gene mutations play a role. The number of cases needing surgical resection is significantly higher for individuals carrying at least one of the three mutations previously described as involved in $\mathrm{CD}$. When each mutation is individually studied, it is observed that G908R and $1007 \mathrm{fs}$ are also more frequent in individuals that have already required surgery. Similar results relate to the specific study of each surgical indication: ileal resection and treatment of fistulae. In both cases, patients carrying one of these mutations show a higher risk of surgical intervention. The fact that the $\mathrm{R} 702 \mathrm{~W}$ mutation is not related to need of surgery confirms the previous observation that this mutation is not a susceptibility marker for $\mathrm{CD}$ in the Galician population (13).

Regarding the higher need for ileal resection in carriers of CARD15 mutations, this had not been previously reported, but is consistent with previous reports of a higher number of mutations in individuals with fibrostenosis, normally with ileal location, who are the ones who usually require surgical resection $(11,12,20)$.

Need for surgery may be considered a signal of severity, and in this respect our results support the observations that the CARD15 gene plays a role in susceptibility and in the severity of disease as previously reported (21-25).

However, it must be noted that despite a relationship between these mutations and the need for CD-related surgery, the percentage of patients carrying these mutations that have also required a surgical intervention is still too low to allow its use in the prediction of disease outcome in this respect. For the time being we suggest that patients with CARD15 mutations have more commonly a severe form of CD requiring surgery. However, studies with larger numbers of patients are indicated to confirm this findings. 


\section{REFERENCES}

1. Gómez de la Concha E, Fernández Franco L. Inflammatory bowel diseases. Relationship between genetics and clinical characteristics. Rev Esp Enferm Dig 2003; 95: 524-6, 521-3.

2. Peña AS, Penate M. Genetic susceptibility and regulation of inflammation in Crohn's disease. Relationship with the innate immune system. Rev Esp Enferm Dig 2002; 94: 524-6, 351-60.

3. Hugot JP, Chamaillard M, Zouali H, et al. Association of NOD2 leucine-rich repeat variants with susceptibility to Crohn's disease. Nature 2001; 411: 599-603.

4. Ogura Y, Bonen DK, Inohara N, et al. A frameshift mutation in NOD2 associated with susceptibility to Crohn's disease. Nature 2001; 411: 603-6.

5. Murillo L, Crusius JB, van Bodegraven AA, et al. CARD15 gene and the classification of Crohn's disease. Immunogenetics 2002; 54: 59-61.

6. Inoue $\mathrm{N}$, Tamura $\mathrm{K}$, Kinouchi $\mathrm{Y}$, et al. Lack of common NOD2 variants in Japanese patients with Crohn's disease. Gastroenterology 2002; 123: 86-91.

7. Yamazaki K, Takazoe M, Tanaka T, et al. Absence of mutation in the NOD2/CARD15 gene among 483 Japanese patients with Crohn's disease. J Hum Genet 2002; 47: 469-72.

8. Croucher PJ, Mascheretti S, Hampe J, et al. Haplotype structure and association to Crohn's disease of CARD15 mutations in two ethnically divergent populations. Eur J Hum Genet 2003; 11: 6-16.

9. Leong RW, Armuzzi A, Ahmad T, et al. NOD2/CARD15 gene polymorphisms and Crohn's disease in the Chinese population. Aliment Pharmacol Ther 2003; 17: 1465-70.

10. Vermeire S, Wild G, Kocher K, et al. CARD15 genetic variation in a Quebec population: prevalence, genotype- phenotype relationship, and haplotype structure. Am J Hum Genet 2002; 71: 74-83.

11. Lesage S, Zouali H, Cezard JP, et al. CARD15/NOD2 mutational analysis and genotype-phenotype correlation in 612 patients with inflammatory bowel disease. Am J Hum Genet 2002; 70: 845-57.

12. Hampe J, Cuthbert A, Croucher PJ, et al. Association between insertion mutation in NOD2 gene and Crohn's disease in German and British populations. Lancet 2001; 357: 1925-8.

13. Núñez C, Barreiro M, Domínguez-Muñoz JE, et al. CARD15 mutations in patients with Crohn's disease in a homogeneous Spanish population. Am J Gastroenterology 2004; 99: 450-6.
14. Larson DW, Pemberton JH. Current concepts and controversies in surgery for IBD. Gastroenterology 2004; 126: 1611-9.

15. Helio $\mathrm{T}$, Halme L, Lappalainen $\mathrm{M}$, et al. CARD15/NOD2 gene variants are associated with familially occurring and complicated forms of Crohn's disease. Gut 2003; 52: 558-62.

16. Vavassori P, Borgiani P, D'Apice MR, et al. 3020insC mutation within the NOD2 gene in Crohn's disease: frequency and association with clinical pattern in an Italian population. Dig Liver Dis 2002; 34: 153.

17. Bairead E, Harmon DL, Curtis AM, et al. Association of NOD2 with Crohn's disease in a homogeneous Irish population. Eur J Hum Genet 2003; 11: 237-44.

18. López Ramos D, Gabriel R, Cantero Perona J, et al. Association of MALTectomy (appendectomy and tonsillectomy) and inflammatory bowel disease: a familial case-control study. Rev Esp Enferm Dig 2001; 93: 303-14.

19. Radford-Smith GL, Edwards JE, Purdie DM, et al. Protective role of appendicectomy on onset and severity of ulcerative colitis and Crohn's disease. Gut. 2002; 51: 808-13.

20. Loftus EV. Clinical epidemiology of inflammatory bowel disease: incidence, prevalence, and environmental influences. Gastroenterology 2004; 126: 1504-17.

21. Buning C, Genschel J, Buhner et al. Mutations in the NOD2/CARD15 gene Crohn's s disease are associated with ileocecal resection and are a risk factor for reoperation. Aliment Pharmacol Ther 2004; 19: 10738.

22. Mendoza JL, Murillo LS, Fernández L, et al. Prevalence of mutations of the NOD2/CARD15 gene and relation to phenotype in Spanish patients with Crohn disease. Scand J Gastroenterol 2003; 38: 123540.

23. Abreu MT, Taylor KD, Lin YC, et al. Mutations in NOD2 are associated with fibrostenosing disease in patients with Crohn's disease. Gastroenterology 2002; 123: 679-88.

24. Buning C, Molnar T, Nagy F, et al. The $1007 \mathrm{fs}$ insC mutation in NOD2/CARD15 in Crohn's disease patients from Hungary: association with stenotic disease behaviour and ileal resections. Gastroenterology. Digestive Disease Week and The 103rd Anual Meeting of the American Gastroenterological Association 2004; 126: 104569(A).

25. Radlmayr M, Torok HP, Martin K, et al. The cinsertion mutation of the NOD2 gene is ssociated with fistulizing and fibrostenotic phenotypes in Crohn's disease. Gastroenterology 2002; 122: 2091-2. 